\title{
Scanning Tunneling Microscopy Images of Transition-Metal Structures Buried Below Noble-Metal Surfaces
}

\author{
S. Heinze, ${ }^{1,2}$ R. Abt, ${ }^{1}$ and S. Blügel ${ }^{1}$ \\ ${ }^{1}$ Institut für Festkörperforschung, Forschungszentrum Jülich, D-52425 Jülich, Germany \\ ${ }^{2}$ Zentrum für Mikrostrukturforschung, Universität Hamburg, D-20355 Hamburg, Germany \\ G. Gilarowski and H. Niehus \\ Institut für Physik, Oberflächenphysik und Atomstoßprozesse, Humboldt-Universität zu Berlin, D-10115 Berlin, Germany
}

(Received 12 August 1999)

\begin{abstract}
We analyze scanning tunneling microscopy (STM) experiments of Ir impurities and chains buried in the subsurface layer of $\mathrm{Cu}(001)$ by first-principles calculations based on the density-functional theory and show for the first time that metal atom defect structures below a metal surface can be detected by STM. We show that this is not particular to the $\mathrm{Ir} / \mathrm{Cu}$ system, but applies to a much wider class of systems, and holds also for defects in deeper layers. For the $\mathrm{Ir} / \mathrm{Cu}$ system we confirm by total energy calculations that the subsurface position is energetically the most favorable one.
\end{abstract}

PACS numbers: 68.35.Bs, 61.16.Ch, 73.20.At, 73.20.Dx

Structural, catalytic, magnetic, and electronic properties of metal surfaces and ultrathin films are strongly altered upon alloying. Novel ordered and disordered alloys are formed at the surface or surface near region due to surface segregation of bulk alloys or upon metal-on-metal growth, where intermixing takes place between deposited and substrate metals, even between metals, which are immiscible in bulk. Over the past few years, the scanning tunneling microscope (STM) developed to a very powerful real space probe providing an unprecedented wealth of new insight into this field [1-8]. It permitted, e.g., the correlation between the surface compositional structure of the alloy on an atomic level and macroscopic properties. Two key issues in this context are (i) the chemical sensitivity of the STM, necessary to discern different chemical components of the alloy, and (ii) the depth sensitivity of the STM, the capability to image alloys below the surface layer or to discern alloy components at different layers below the surface. While it is common knowledge that chemical sensitivity can quite often be achieved, no evidence for imaging more than the surface layer exists although subsurface growth of $\mathrm{Pd}$ on $\mathrm{Cu}(110)$ and $\mathrm{Ag}(110)$ has been studied by STM [6]. This seems to be in accordance with the conventional wisdom of effective screening of impurities below the surface by the nearly free electrons of the metals and that the STM tip follows the surface topography of surface atoms. In the case of semiconductors screening is weaker and imaging even of uncharged impurities down to the third layer below the surface has been reported [9].

In the present work we demonstrate for the first time that transition-metal structures buried below a metal surface can be imaged by STM. We combine STM, ion scattering spectroscopy (ISS), x-ray photoelectron spectroscopy (XPS), and low-energy electron diffraction (LEED) experiments with $a b$ initio calculations and show this explicitly for the subsurface alloy formed after deposition of $\mathrm{Ir}$ on $\mathrm{Cu}(001)$. We provide arguments and results showing that this is not particular to the $\mathrm{Ir} / \mathrm{Cu}(001)$ system but applies to a wide class of transition metals buried in noble-metal substrates.

STM images were taken at room temperature with an OMICRON combined STM and atomic force microscope (AFM) operated in constant-current mode. Figure 1(a) shows a typical STM image with atomic resolution for Ir deposited on a $\mathrm{Cu}(001)$ surface at low coverage. It exhibits a regular array of white dots and reveals disordered starlike defects as depressions. As carefully checked in the present work no bias-voltage-dependent corrugation reversal occurs on $\mathrm{Cu}(001)$ and the regular array of dots is interpreted as $\mathrm{Cu}$ atoms. The apparent depth of the starlike depressions is about $0.3 \AA$ and is assigned to single Ir atoms. Note that the center of gravity of these defects is not located on regular $\mathrm{Cu}$ sites in the surface lattice, but instead at the fourfold-hollow sites. Excluding interstitial positions for Ir due to the large radius of the atom, Ir must be located below or on top
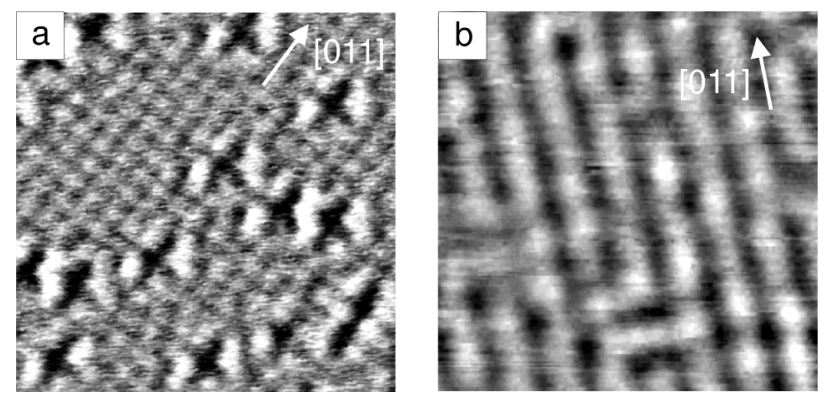

FIG. 1. STM images of the Cu-Ir surface: (a) Low Ir coverage. $0.05 \mathrm{ML}$ of Ir deposited at $200 \mathrm{~K}$ and annealed at $650 \mathrm{~K}$ $\left(U_{\text {sample }}=0.02 \mathrm{~V}, I=2.1 \mathrm{nA}\right)$. (b) Ordered $\mathrm{CuIr} / \mathrm{Cu}(100)$ subsurface alloy. $0.6 \mathrm{ML}$ of $\mathrm{Ir}$ deposited at $620 \mathrm{~K}\left(U_{\text {sample }}=\right.$ $-0.3 \mathrm{~V}, I=0.5 \mathrm{nA})$. Image sizes: $40 \AA \times 40 \AA$. Tip-tosample distance $z \approx 6-9 \AA$. 
of the surface. The latter can be discarded on the basis of $\mathrm{He}^{+}$ISS measurements, which are known to be sensitive to the topmost surface layer only [10]. No Ir signal was detected by ISS.

A closer inspection of the image further reveals that sometimes the starlike defects overlap when two Ir atoms are located at nearest-neighbor sites. As a consequence dark lines start to form along the [011] directions [cf. dark line in the lower right part of Fig. 1(a)]. After direct deposition of Ir [0.5-0.6 monolayer (ML)] at elevated temperatures long range ordering occurs which is indicated by a $(2 \times 1)$ LEED superstructure exposing two domains. The amount of Ir visible in ISS is then below 1\%, whereas XPS results show that Ir is segregated into the surface but remains in a surface near region, e.g., in the second layer. The corresponding STM image of the ordered surface exhibits a distinct chainlike structure orientated along the two equivalent [011] directions [Fig. 1(b)]. The distance between adjacent chains of the same kind is measured to about $5 \AA$ (i.e., twice the nearest-neighbor distance) in agreement with a $(2 \times 1)$ LEED structure. The ordered alloy shows an apparent corrugation of $0.3 \AA$ as measured by STM. Successful imaging of the subsurface alloy was possible only for negative bias voltages $U_{\text {sample }}$ (occupied sample states) in a range between -0.2 and $-0.5 \mathrm{~V}$, indicating electronic effects as the origin of the measured corrugation. Summarizing the experimental findings from the STM, LEED, ISS, and XPS measurements we propose the following structural model: $0.5 \mathrm{ML}$ of Ir deposited on $\mathrm{Cu}(001)$ leads to the formation of an ordered $(2 \times 1) \mathrm{CuIr}$ alloy layer of adjacent $\mathrm{Cu}$ and $\mathrm{Ir}$ chains along the [011] directions buried under one monolayer of $\mathrm{Cu}$.

Although above experimental findings lead to a consistent structural model of an ordered CuIr subsurface alloy, (i) the result is quite surprising as $\mathrm{Ir}$ and $\mathrm{Cu}$ exhibits a large miscibility gap in the bulk phase diagram [11], and (ii) the model relies on the assumption that STM is able to image Ir impurities and chains buried in the substrate, for which there is no experimental evidence so far and disputed by the conventional school of thought that STM follows the topography of surface atoms. In order to shed light onto the question of detectibility of buried Ir structures, and to interpret and understand STM images of Ir point defects and CuIr adjacent chains, we calculated STM images on the basis of the model of Tersoff and Hamann [12] and first-principles electronic structure calculations.

Electronic structure, total energy, and force calculations are carried out with the full-potential linearized augmented plane wave method in film geometry [13] within the local-density approximation [14] to the densityfunctional theory. The surface and surface near region are modeled by eleven-layer (001) films, at the experimental lattice constant of $\mathrm{Cu}\left(a_{\mathrm{Cu}}=3.616 \AA\right)$, consisting of nine layers of $\mathrm{Cu}$ and one layer containing $\mathrm{Cu}$ and $\mathrm{Ir}$ atoms placed on both sides of the film at the surface $(S)$, sub- surface $(S-1)$, or deeper $(S-2, S-3)$ layers. The Ir impurity is modeled using a $p(2 \times 2)$ surface unit cell containing three $\mathrm{Cu}$ atoms and one $\mathrm{Ir}$ atom per impurity layer. Similarly the CuIr chain structure is treated in a $p(2 \times 1)$ unit cell with two atoms per layer. For the system with the $p(2 \times 1)$-CuIr chains at $S-1$ all atom positions are fully relaxed, minimizing the total energy by force calculations. We found a buckling of the Ir and $\mathrm{Cu}$ atoms at the $S-1$ layer of $\Delta Z / d=2.9 \%$ of the interlayer distance $d=a_{\mathrm{Cu}} / 2$ between two (001) plains. This increases the corrugation amplitudes of STM images presented below by $\Delta z \simeq 0.05 \AA$, which is a minor contribution in the context of this Letter and the relaxation is not considered any further. We further investigated the energetic stability of the subsurface alloy and the energetic competition with a checkerboard arrangement of $\mathrm{Cu}$ and Ir atoms by total energy calculations. We found, consistent with the interpretation of the STM analysis, Ir in the $p(2 \times 1)$ CuIr structure located in the subsurface layer is the energetically most stable configuration, $49 \mathrm{meV}$ lower than located at deeper layers.

The tunneling current $I$ that gives rise to the STM images is a function of the applied bias voltage $U$ and is given by

$$
I\left(\mathbf{r}_{\|}, z, U\right) \propto \int_{-\infty}^{+\infty} g_{U, T}(\epsilon) n\left(\mathbf{r}_{\|}, z, \epsilon_{F}+\epsilon\right) d \epsilon,
$$

where $\epsilon_{F}$ is the Fermi energy, $n\left(\mathbf{r}_{\|}, z, \epsilon_{F}+\boldsymbol{\epsilon}\right)$ is the local density of states (LDOS) of the sample evaluated at the lateral $\left(\mathbf{r}_{\|}\right)$and vertical $(z)$ [15] positions of the tip. $f$ is the Fermi function $g_{U, T}$ is the difference of the Fermi function $f_{T}$ at $\epsilon_{F}-e U+\epsilon$ and $\epsilon_{F}+\epsilon$. All calculations are carried out for room temperature, $k_{B} T=0.025 \mathrm{eV}$. The corrugation amplitude $\Delta z$, i.e., the maximum variation in the vertical position of the tip as it scans the surface at constant current, has been calculated as described in Ref. [16]:

$$
\Delta z(z, U) \propto \frac{\int g_{U, T}(\epsilon) n_{2}(z, \epsilon) d \epsilon}{\int g_{U, T}(\epsilon)(\partial / \partial z) n_{1}(z, \epsilon) d \epsilon},
$$

where $n_{1,2}$ are the lowest order LDOS star coefficients. These star coefficients are obtained expanding the LDOS in symmetrized 2D plane waves ("star" functions $\phi_{s}$ ) with $z$-dependent coefficients:

$$
n\left(\mathbf{r}_{\|}, z, \epsilon\right)=\sum_{s} n_{s}(z, \epsilon) \phi_{s}\left(\mathbf{r}_{\|}\right) .
$$

The first star function $\phi_{1}$ is simply a constant and does not contribute to the corrugation pattern. Its coefficient $n_{1}$ must be positive since it represents the charge integrated over the 2D unit cell. $n_{2}$ can be positive or negative and determines the sign of the corrugation amplitude and thus the chemical and structural sensitivity.

The theoretically determined STM images for the Ir impurity $[p(2 \times 2)]$ and chain $[p(2 \times 1)]$ structure buried in the subsurface layer $S-1$ (Fig. 2) calculated 


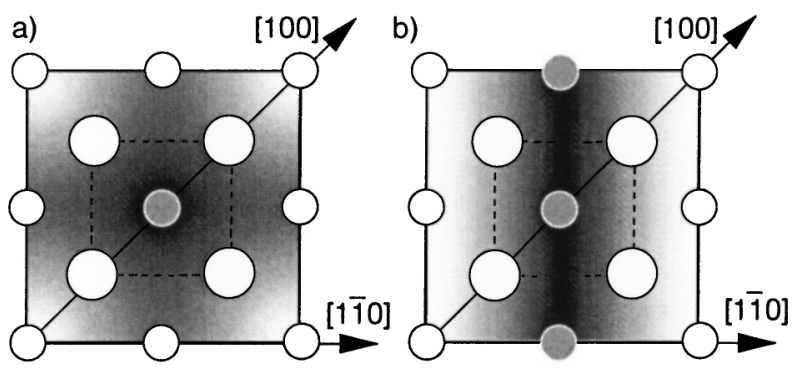

FIG. 2. Calculated STM images at $U=-0.6 \mathrm{~V}, z=5 \AA$ for Ir impurity (a) and chain (b) located at $S-1$. Open (full) circles represent $\mathrm{Cu}$ (Ir) atoms; big (small) circles represent atoms at $S(S-1)$.

for the bias voltage $U=-0.6 \mathrm{~V}$ clearly reproduces the experimental images of star- and chainlike patterns.

A wide range of the bias-voltage dependence of STM images is covered in Fig. 3 in terms of calculated corrugation amplitudes for Ir impurities and chains at the $\mathrm{Cu}(001)$ surface layer $S$ or buried in the subsurface layer $S-1$. From Fig. 3 it can be concluded that Ir atoms and chains in the surface should be imaged as protrusions $(\Delta z>$ $0)$ while depressions $(\Delta z<0)$ should be found for the buried structures, and vice versa for $\mathrm{Cu}$ atoms. Strikingly the absolute corrugation amplitudes are of comparable values, irrespective whether Ir is in the surface or subsurface layer. Note the distinct bias-voltage dependence for the buried structures. The maximum corrugation amplitudes for the buried structures were found around $-0.5 \mathrm{eV}$ and an atomic resolution becomes possible only at negative bias voltages, i.e., occupied sample states, which is in perfect agreement with the experimental observation.

Further calculations for Ir impurities and chains at deeper layers (not shown here) reveal that the maximum corrugation amplitude decreases for the Ir impurity with increasing depth (i.e., $\Delta z=0.15 \AA$ at $S-2$ ), but re-

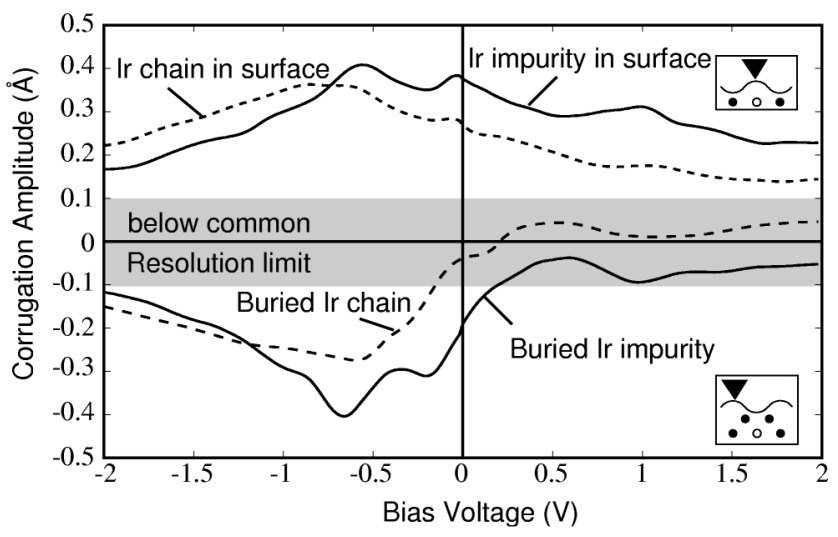

FIG. 3. Calculated corrugation amplitudes of a tip at $z=$ $5.3 \AA$, as a function of the applied bias voltage $U$ for the $\mathrm{Ir}$ impurity and chain. In the insets at the upper and lower right corners, filled (open) circles denote $\mathrm{Cu}$ (Ir) atoms. Positive (negative) corrugation amplitudes are defined as imaging the Ir site as a protrusion (depression). mains nearly unchanged for the CuIr chain structure. The latter is a clear indication of the development of quantumwell states between the $\mathrm{Cu}$ surface and the buried structure analogous to the work of Altfeder et al. [17], absent for impurities. Since the corrugation amplitudes for $\mathrm{CuIr}$ chains buried at different layers are very similar, and from Fig. 1(b) it is a priori unknown whether $\mathrm{Cu}$ or $\mathrm{Ir}$ is imaged as protrusion it is impossible to conclude from Fig. 1(b) at which layer the CuIr alloy is located. On the other hand, in the low coverage limit, single defects [Fig. 1(a)] are clearly related to single Ir atoms. Since they appear as depressions with a similar corrugation amplitude as the chains we conclude that Ir is located in the subsurface layer.

In order to correlate the bias-voltage dependence of the corrugation amplitudes to the underlying electronic structure we present in Fig. 4 , for the $p(2 \times 1)$-CuIr chain structure buried in $S-1$, the second star coefficient of the vacuum LDOS. We recall from the discussion of Eq. (2) that the corrugation pattern is determined by $n_{2}$. For a perfect $\mathrm{Cu}(001)$ surface, the two $\mathrm{Cu}$ atoms in a $p(2 \times 1)$ surface cell are indistinguishable, the difference in the corrugation between $\mathrm{Cu}$ atom one and two is zero, and thus $n_{2}$ is identically zero. Replacing one chain of $\mathrm{Cu}$ atoms by $\mathrm{Ir}$ atoms breaks the symmetry and $n_{2}$ will be nonzero. In Fig. 4 we find $n_{2}$ is basically zero over a large energy range, except around $-0.5 \mathrm{eV}$, where $n_{2}$ shows a negative peak. According to our definition, a negative value of $n_{2}$ means Ir is imaged as depression and $\mathrm{Cu}$ as protrusion. The fact that $n_{2}$ is zero for positive bias voltages explains why no atomic resolution was found in the experiment at $U>0$.

The peak of $n_{2}$ is a consequence of electronic states spread over a large part of the 2D Brillouin zone (BZ). For the Ir chain system buried at $S-1$, a real space view of a typical state at the energy of the peak of $n_{2}$ is shown in Fig. 5 in terms of a charge density contour plot. Figure 5 displays the fact that the hybridization of the Ir $d$-states (largest charge density in Fig. 5) with the $\mathrm{Cu} s p$ states results in tilted $p d$ orbitals at the $\mathrm{Cu}$ surface atoms (and of the $\mathrm{Cu}$ atoms below the Ir atoms, $S-2$ ). The bond between the $\mathrm{Cu}$ surface atom and the $\mathrm{Ir}$ atom is of antibonding nature and the tilted $p d$ orbitals form a bond charge above the $\mathrm{Cu}$ subsurface atom, which implies

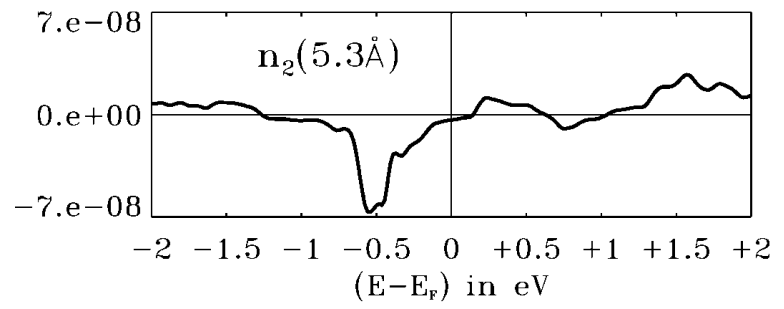

FIG. 4. LDOS star coefficient $n_{2}(z=5.3 \AA)$ as a function of energy for the CuIr chain structure buried at $S-1$. 


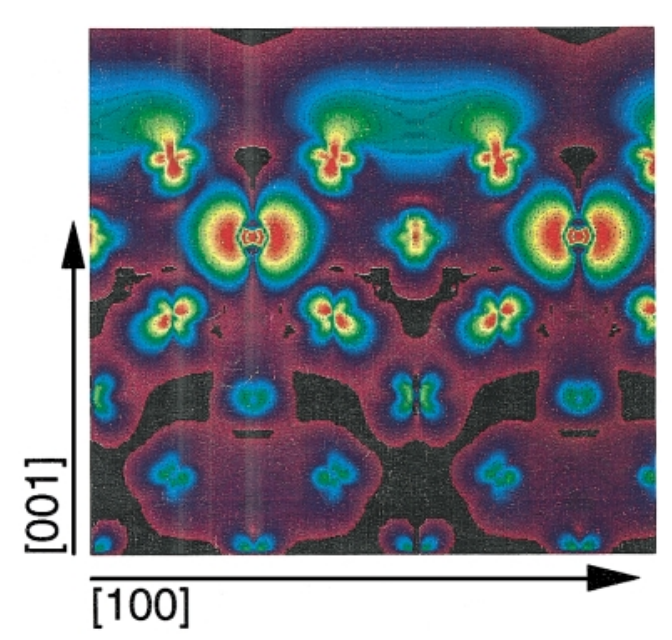

FIG. 5 (color). Cross section along the [100] direction through the charge density of a typical state $\left[\mathbf{k}_{\|}=\right.$ $(\pi / a)(0.325,0.325)]$ in the $2 \mathrm{D} \mathrm{BZ}$ at an energy $E_{F}-0.6 \mathrm{eV}$ for Ir chains at $S-1$.

that the charge density maximum, which is for a plain $\mathrm{Cu}(001)$ surface above the $\mathrm{Cu}$ surface atom, shifts to the position above the $\mathrm{Cu}$ subsurface atom. Depending on the structural symmetry this results in the starlike or stripelike pattern observed in experiment (Fig. 1) and theory (Fig. 2) for the CuIr impurity and chain structure at $S-1$.

The arguments based on the hybridization of the $\mathrm{Cu}$ $s p$-states and $\operatorname{Ir} d$-states are rather general and the visibility of buried transition-metal atoms should also hold for other transition-metal atoms. First candidate is $\mathrm{Rh}$, isoelectronic to Ir. With an increasing number of $d$ electrons ( $\mathrm{Ir}, \mathrm{Pt}, \mathrm{Au})$ we expect that the $d$-band energy lowers with respect to the Fermi energy and thus the tunneling barrier of the state seen in STM becomes higher. This increases the decay of the wave function into the vacuum and the corrugation amplitudes should drop. In addition a lower $d$-band energy reduces the symmetry breaking between $\mathrm{Cu}$ and the $3 d$ metal, $n_{2}$ approaches zero, and again the corrugation amplitudes should drop. For elements with a decreasing number of $d$ electrons (Ir, Os, Re, ...), the opposite trend is expected. We confirmed this picture by carrying out calculations for Ta, $\mathrm{W}, \mathrm{Re}, \mathrm{Os}, \mathrm{Pt}$, and $\mathrm{Ag}$ chains in $\mathrm{Cu}(001)$ at $S-1$. For $\mathrm{Pt}$, the corrugation amplitude drops to a maximum value of $0.15 \AA$ at $U=-1.2 \mathrm{~V}$ and an amplitude lower than $0.05 \AA$ within an energy of $U= \pm 0.5 \mathrm{~V}$. We conclude it is most likely impossible to image buried Pt. We speculate that this is the reason why the subsurface growth of $\mathrm{Pd}$, isoelectronic to $\mathrm{Pt}$, in $\mathrm{Cu}(110)$ [6] was impossible to image with STM. For Ag buried in $\mathrm{Cu}(001)$, we found corrugation amplitudes of $0.03 \AA$, which will hardly be measurable by STM. For Os, Re, W, and Ta, on the other hand, corrugation amplitudes of $\sim 0.4 \AA$ are found in a bias-voltage interval of $U= \pm 0.5 \mathrm{~V}$. The maximum corrugation amplitude increases in energy with a decreasing number of $d$ electrons and the buried Ta chains can be detected only for $U>0$.

In conclusion, we have given experimental and theoretical evidence that it is possible to image transition-metal atoms buried in noble-metal surfaces by STM. The corrugation amplitudes of alloys at the surface or buried can be of similar size and STM experiments in combination with further surface sensitive tools (e.g., ISS) are required to draw firm conclusions on the expected site below the surface. This should not only hold for the Ir/Cu system, but is a rather general phenomenon expected for a large class of transition metal systems.

This work was supported by the DFG under Grants No. BL444/1-1 and No. WI1277/6-1, by the Program "Verbundforschung Synchrotronstrahlung" of the BMBF, and the TMR Networks, Contracts No. EMRX-CT960089 and No. FMRX-CT98-0178. G. G. and H. N. gratefully acknowledge the financial support by the DFG via Sfb 290 TP B7.

[1] D. D. Chambliss and S. Chiang, Surf. Sci. Lett. 264, L187 (1992).

[2] M. Schmid, H. Stadler, and P. Varga, Phys. Rev. Lett. 70, 1441 (1993).

[3] L. P. Nielsen et al., Phys. Rev. Lett. 71, 754 (1993).

[4] H. Röder, R. Schuster, H. Brune, and K. Kern, Phys. Rev. Lett. 71, 2086 (1993).

[5] P.W. Murray, I. Stensgaard, E. Lægsgaard, and F. Besenbacher, Phys. Rev. B 52, R14 404 (1995).

[6] P. W. Murray et al., Phys. Rev. B 55, 1380 (1997).

[7] A. Davies, J. A. Stroscio, D. T. Pierce, and R. J. Celotta, Phys. Rev. Lett. 76, 4175 (1996).

[8] P. T. Wouda et al., Surf. Sci. 359, 17 (1996).

[9] For a review, see Ph. Ebert, Surf. Sci. Rep. 33, 121 (1999).

[10] H. Niehus, W. Heiland, and E. Taglauer, Surf. Sci. Rep. 17, 578 (1993).

[11] Binary Alloy Phase Diagrams, edited by T. B. Massalski (AMS International, Materials Park, OH, 1990).

[12] J. Tersoff and D. R. Hamann, Phys. Rev. Lett. 50, 1998 (1983).

[13] E. Wimmer, H. Krakauer, M. Weinert, and A. J. Freeman, Phys. Rev. B 24, 864 (1981); M. Weinert, E. Wimmer, and A. J. Freeman, ibid. 26, 4571 (1982).

[14] S. H. Vosko, L. Wilk, and N. Nusair, Can. J. Phys. 58, 1200 (1980).

[15] In this paper the vertical distance $z$ is always measured between the center of the surface and the tip atom (nucleus-nucleus distance).

[16] S. Heinze et al., Phys. Rev. B 58, 16432 (1998).

[17] I. B. Altfeder, D. M. Chen, and K. A. Matveev, Phys. Rev. Lett. 80, 4895 (1998). 